\title{
The Correlation of $\gamma$-Glutamyl Transferase $(\gamma$-GT), Glutathione Peroxidase (GPx) and Total Antioxidant Status (TAS) with Inflammatory Marker in Individuals with Metabolic Syndrome
}

\author{
Susana Gunawan ${ }^{1,2^{*}}$, Anwar Santoso $^{3}$, Andi Wijaya ${ }^{1,4}$
}

\author{
${ }^{1}$ Post Graduate Program in Clinical Biochemistry, Hasanuddin University, Jl. Perintis Kemerdekaan Kav.10, Makassar, Indonesia \\ ${ }^{2}$ Prodia Clinical Laboratory, Jl. Diponegoro 192, Denpasar, Indonesia \\ ${ }^{3}$ National Cardiovascular Center-Harapan Kita, Jl. Letjen. S. Parman Kav 87, Slipi, Jakarta, Indonesia \\ ${ }^{4}$ Prodia Clinical Laboratory, JI. Kramat Raya 150, Jakarta, Indonesia \\ *Correspondence: susana_gunawan@prodia.co.id
}

\section{Abstract}

$\mathrm{B}$ ACKGROUND: $\gamma$-GT is known as a primary enzyme that constituent extracellular glutathione catabolism. Various researches have proven that an increase in $\gamma$-GT concentration is related to oxidative stress and to components of metabolic syndrome. With individuals having metabolic syndrome as research subjects, our objective is to find the correlation of $\gamma$-GT, GPx and SAT.

METHODS: This was a cross sectional study done on 36 subjects with metabolic syndrome who met the IDF 2005 criteria. Statistical analysis was carried out using SPSS for Windows ver. 11.5 with a significance level of $\mathrm{p}<0.05$. The correlation among biomarkers were assessed using Spearman's Rho test.

RESULTS: $\gamma$-GT concentration was correlated negatively with GPx concentration $(r=-0.411, p=0,014)$, but correlated positively with hs-CRP concentration $(\mathrm{r}=$ $0.385, p=0,022)$. The increase of $\gamma$-GT concentration had a potential risk 8.5 times higher than the increase of hs-CRP concentration, which proved to be more dominant in comparison to its risk to $\gamma$-GT/GPx or $\gamma$-GT/SAT ratios $(\mathrm{p}<0.05)$.

CONCLUSIONS: An increase in $\gamma$-GT concentration characterizes signs of oxidative stress syndrome and of inflammation in individuals with metabolic syndrome.

KEYWORDS: $\gamma$-GT, GPx, SAT, Inflammation, Metabolic Syndrome.

Indones Biomed J 2011; 3(1): 57-63

\section{Introduction}

Metabolic syndrome is categorized as a stage of subclinical inflammation and oxidative stress. Oxidative stress is resulted from Reactive Oxigen Species (ROS) oxidizing macro molecules and damaged cells. Gamma glutamyltransferase $(\gamma$-GT) enzyme indirectly causes oxidative stress since ROS is released from redox reaction, in which $\gamma$-GT has a crucial role in its process (1). In another study, Giral, et al. reported that $27.6 \%$ of dislipidemic patients experienced an increase in hepatic transaminase and $\gamma$-GT, which were significantly related to the component of metabolic syndrome $(2,3)$.

In clinical practice, $\gamma$-GT serum has been recognized as a diagnostic marker for liver function, and also as a strong predictor of cardiovascular disease and heart failure-related death $(4,5)$. Another study conducted by Giral, et al. on a different group of patients also found similar results. They noted that in metabolic syndrome and 
dyslipidemic patients, an increase of $\gamma$-GT concentration was strongly related to prooxidant thiol group and its component. There was a decrease in plasma glutathione level and an increase in cysteinyl-glycine (CysGly) and cysteine. This imbalanced condition presented oxidative stress, hence a cardiovascular risk factor (2).

Extracellular glutathione broken down by $\gamma$-GT triggers release of ROS through a chemical reaction on CysGly (6). CysGly is a strong reduction substance for $\mathrm{Fe}^{3+}$ at the extracellular space, which increases the production of $\mathrm{Fe}^{2+}$ and free radical Thiol level. Further reaction of this reduction constitutes an increase in production of radical anion superoxidant. By examining Total Antioxidant Status (TAS), we are able to measure the total integration of the body's antioxidant system that represents every biological component with its antioxidant properties, especially at the extracellular space. This clinical examination is used for various purposes such as to see antioxidant deficiency risk in patients with certain diseases and to use as a gauge in optimizing antioxidant therapy.

Based on results of other studies done previously, our present study had the main purpose of assessing the correlation of Gamma-Glutamyl Transferase, Glutathione Peroxidase and Total Oxidant Status with Inflammatory Incident in individuals with metabolic sydrome.

\section{Methods}

\section{STUDY SUBJECTS}

The study subjects were males and females who had a health check up at Prodia Clinical Laboratory of Denpasar and Malang branches. Each study subject was asked to fill in a questionnaire that covered information of the subject's medical treatment history, smoking habit, alcohol drinking habit, health supplement consumption, and lifestyle. Physical examinations done in this study included measurements of blood pressure, waist circumference, weight and height. The criteria for Metabolic syndrome used were in compliance with IDF 2005, where waist measurement $>90 \mathrm{~cm}$ (male) and $>80 \mathrm{~cm}$ (female) and fulfilling at least 2 out of 4 other criteria: hypertriglyceride $(\geq 150 \mathrm{mg} / \mathrm{dL}$ or on going treatement), HDL $<40 \mathrm{mg} / \mathrm{dL}$ (male) or HDL $<50 \mathrm{mg} / \mathrm{dL}$ (female), glucose level increase ( $\geq 100 \mathrm{mg} / \mathrm{dL}$ or on going treatement), hypertension $(\geq$ $130 / 85 \mathrm{mmHg}$ ).

Exclusion criteria for this study complied to patients with hepatitis, fatty liver, cancer, cirrhosis, and habit of smoking and alcohol drinking. An informed consent was obtained from each study subject prior to collection of samples. This study was approved by Komite Etik Universitas Hasanuddin (Number 0180/H04.8.4.5.31/ PP36-KOMETIK/2010). There were 36 patients (25 males, 11 females) recruited in the study who had met all the criteria listed above.

\section{BLOOD SAMPLING AND ANALYTIC METHOD}

Blood samples were taken from the patients' veins within a time period from 8:00 am to 10:00 am. The patients went through a 12 hour period of fasting starting right before collection of blood samples. The blood samples were frozen for 30 minutes and then centrifuged at $3500 \mathrm{rpm}$ for 15 minutes. Sera were then isolated and aliquoted. Examination of $\gamma$-GT was done with automatic Modular P800 with Roche reagent kit, whereas hsCRP was measured using Immulite 2000 with DPC reagent kit. TAS and GPx parameters were processed by colorimetry method using Randox Laboratories reagent kit. $\gamma$-GT reference range: 8 $-61 \mathrm{U} / \mathrm{L}$ (male) and $7-32 \mathrm{U} / \mathrm{L}$ (female). hsCRP reference range: $<10 \mathrm{mg} / \mathrm{L}$. GPx reference range: $27.5-73.6 \mathrm{U} /$ gHb. TAS reference range: $1.30-1.77 \mathrm{mmol} / \mathrm{L}$. Reference ranges for hsCRP, GPx and SAT were identical for both male and female patients.

\section{DATA ANALYSIS}

Data analyses were done using SPSS 11.5 statistical analysis software for windows (SPSS inc., Chicago,IL,USA). Distribution of continuous variables were assessed for normality using Kolmogorov-Smirnov. Associations between variables were analyzed using Spearman's correlation analysis with significance level at $\mathrm{p}<0.05$. We also used regression analysis between variables.

\section{Results}

This research was done in Prodia Clinical Laboratory in Denpasar and Malang, and Prodia National Reference Laboratory in Jakarta, in the period from 13 February to 5 March 2010. The study samples were taken from 36 patients with an average age of 48 years and gender composition of 11 or $30.6 \%$ females and 25 or $69.4 \%$ males.

In order to have the basic analysis for this study, early analysis was done by examining the positivity and negativity of $\gamma$-GT correlation with GPx, -CRP and TAS individually. And beyond its correlation polarity, the early stage of the study examined its correlation significance. 
Table 1. Descriptive Data on Research Objects

\begin{tabular}{|c|c|c|c|c|c|}
\hline Variable & & Median & Mean \pm SD & Min & Max \\
\hline \multirow[t]{2}{*}{ Age (Years) } & Male & 44.0 & $45.0 \pm 7.8$ & 31.0 & 58.0 \\
\hline & Female & 56.0 & $55.0 \pm 4.9$ & 45.0 & 60.0 \\
\hline \multirow[t]{2}{*}{ Weight (kg) } & Male & 79.0 & $81.0 \pm 8.1$ & 66.0 & 96.0 \\
\hline & Female & 60.0 & $63.0 \pm 8.4$ & 52.0 & 79.0 \\
\hline \multirow[t]{2}{*}{ Waist (cm) } & Male & 99.09 & $9.0 \pm 5.4$ & 91.0 & 111.0 \\
\hline & Female & 91.0 & $91.0 \pm 6.7$ & 82.0 & 105.0 \\
\hline \multirow[t]{2}{*}{ Systolic Pressure (mmHg) } & Male & 120.0 & $127.0 \pm 18.4$ & 100.0 & 170.0 \\
\hline & Female & 125.0 & $129.0 \pm 12.3$ & 113.0 & 150.0 \\
\hline \multirow[t]{2}{*}{ Diastolic Pressure (mmHg) } & Male & 90.0 & $83.0 \pm 12.2$ & 60.0 & 110.0 \\
\hline & Female & 80.0 & $82.0 \pm 6.4$ & 70.0 & 90.0 \\
\hline \multirow[t]{2}{*}{ FPG (mg/dL) } & Male & 87.0 & $94.5 \pm 20.2$ & 76.0 & 148.0 \\
\hline & Female & 92.0 & $07.4 \pm 32.1$ & 71.0 & 163.0 \\
\hline \multirow[t]{2}{*}{$\mathrm{HDL}(\mathrm{mg} / \mathrm{dL})$} & Male & 36.0 & $36.4 \pm 5.2$ & 25.0 & 48.0 \\
\hline & Female & 47.0 & $49.7 \pm 9.3$ & 34.0 & 64.0 \\
\hline \multirow[t]{2}{*}{ TG (mg/dL) } & Male & 193.0 & $231.9 \pm 100.7$ & 115.0 & 546.0 \\
\hline & Female & 183.0 & $226.8 \pm 99.4$ & 150.0 & 479.0 \\
\hline \multirow[t]{2}{*}{ V-GT (U/L) } & Male & 31.0 & $40.1 \pm 27.7$ & 17.0 & 123.0 \\
\hline & Female & 29.0 & $48.5 \pm 62.6$ & 13.0 & 223.0 \\
\hline \multirow[t]{2}{*}{ hsCRP (mg/L) } & Male & 1.0 & $1.8 \pm 1.56$ & 0.3 & 6.9 \\
\hline & Female & 1.3 & $2.1 \pm 1.66$ & 0.4 & 5.5 \\
\hline \multirow[t]{2}{*}{ GPx (U/gHb) } & Male & 43.1 & $46.7 \pm 11.4$ & 31.7 & 83.1 \\
\hline & Female & 41.3 & $46.9 \pm 12.6$ & 28.1 & 64.7 \\
\hline \multirow[t]{2}{*}{ TAS (mmol/L) } & Male & 1.9 & $2.0 \pm 1.92$ & 1.6 & 2.37 \\
\hline & Female & 2.0 & $2.0 \pm 1.14$ & 1.6 & 2.21 \\
\hline
\end{tabular}

Notes: FPG = Fasting Plasma Glucose HDL = Cholesterol HDL; TG = Triglyceride; Min = Minimum; Max = maximum. 
Table 2. Correlation Data of y-GT with GPx, SAT and hsCRP

\begin{tabular}{lccc}
\hline Variable & \multicolumn{1}{c}{ v-GT } & $\mathbf{p}$ \\
\hline GPX & $\mathbf{r}$ & 0.014 \\
TAS & $-0.411^{*}$ & 0.150 \\
hSCRP & -0.249 & 0.022 \\
\hline
\end{tabular}

Based on the previous data obtained, we continued to do a regression analysis. The analytical results are shown in Table 3 on odds ratio of $\gamma$-GT to GPx of 0.125 , indicating that the patients who experienced increase of $\gamma$-GT concentration would have a decrease in GPx content. It is known that GPx has a protective factor on $\gamma$-GT. The analytical data also showed a $\gamma$-GT to hsCRP odds ratio of 8.5. This finding indicated that an increase in $\gamma$-GT concentration would cause an 8.5 times increase in the concentration of hsCRP.

The findings that $\gamma$-GT had a negative correlation with
GPx and TAS in the early stage of this study rendered us to do a further analysis on the correlation of $\gamma$-GT/GPx and $\gamma$-GT/TAS ratio with hsCRP. The analysis results showed a positive correlation and significant ratio $\gamma$-GT/GPx with $\operatorname{hsCRP}(\mathrm{r}=0.123, \mathrm{p}=0.025)$ and positive correlation and insignificant ratio $\gamma$-GT/TAS with hsCRP $(r=0.331, p=$ 0.052).

An increase of $\gamma$-GT/GPx ratio also caused the risk of increase of hsCRP concentration with an odd ratio of 7.143 , while $\gamma$-GT/TAS ratio had the risk of increase of hsCRP concentration with an odds ratio of 4.821 .

Table 3. Regression Analysis y-GT, GPx, TAS and hs CRP

\begin{tabular}{|c|c|c|c|c|c|c|c|}
\hline \multicolumn{2}{|c|}{ Variables } & \multirow{2}{*}{\multicolumn{2}{|c|}{$r / p$}} & \multirow[t]{2}{*}{ Odd ratio } & \multirow[t]{2}{*}{$\mathbf{P}$} & \multicolumn{2}{|c|}{$95 \%$ C.I for EXP (B) } \\
\hline & & & & & & Lower & Upper \\
\hline \multirow[t]{3}{*}{ V-GT } & GPx & $-0.461^{* *} /$ & 0.005 & 0.125 & 0.009 & 0.026 & 0.593 \\
\hline & TAS & $-0.249 /$ & 0.150 & 0.500 & 0.313 & 0.130 & 1.923 \\
\hline & hsCRP & $0.482^{* *} /$ & 0.003 & 8.500 & 0.006 & 1.842 & 39.227 \\
\hline Y-GT/GPx & hsCRP & $0.379 * \quad /$ & 0.025 & 7.143 & 0.014 & 1.484 & 34.384 \\
\hline $\mathrm{Y}$-GT/TAS & & $0.331 /$ & 0.052 & 4.821 & 0.037 & 1.097 & 21.192 \\
\hline
\end{tabular}

This study proceeded to evaluate the correlation of the number of metabolic syndrome components with $\gamma$-GT, GPx, TAS and hsCRP. The results showed there was a correlation of the number of metabolic syndrome components with $\gamma$-GT, GPx, TAS and hsCRP, although not significant. Whether there were patients with 2 metabolic syndrome components or patients with 3 metabolic syndrome components, the study results showed no significant corellation with $\gamma$-GT, TAS and hsCRP. However, when a correlation analysis on the number of metabolic syndrome components with GPx content was done, we found a significant correlation with $\mathrm{r}=0.343(\mathrm{p}$ $=0.05$ ). 


\section{Discussions}

Various cohort studies have proven that $\gamma$-GT plasma concentration correlated positively with severity of coronary heart disease, complication risks, and death from cardiovascular disease $(7,8)$. Studies done on a number of patients with dyslipidemia and metabolic syndrome have shown an increase in $\gamma$-GT enzyme activity. This increase in $\gamma$-GT was considered to be related with the potential condition of pro-oxidant components of thiol compounds, where a decrease in glutathione plasma level and an increase in cysteine-glycine occured (9). Relationship with the increase in $\gamma$-GT activity was stronger if the patient sufferred 4 to 5 number of metabolic syndrome components (NCEP-ATP criteria III). An increase in $\gamma$-GT activity is perceived to be related with insuline resistance, central obesity as well as with hepatic steatosis. Hence, an increase in $\gamma$-GT activity level reflects hepatic steatosis and presents oxidative stress condition (2).

$\gamma$-GT's role in increasing risk factor for cardiovascular disease in metabolic syndrome is associated with the increase in CysGly and cysteine when $\gamma$-GT activity level arises as a compensation of the decrease in glutathione level (2). $\gamma$-GT concentration detected in the serum is suspected to be the cause of cell regeneration process and cellular stress condition (10). $\gamma$-GT enzyme eliminates $\gamma$-glutamyl linkages from glutathione after CysGly is broken down by dipeptidase membrane in glutathione metabolism process. Loose cysteine is then brought to the cell and used as a substrate in de novo GSH synthesis. This $\gamma$-glutamyl cycle is important to limit GSH cellular quantity (11).

In this study we found that an increase in $\gamma$-GT concentration was related to GPx content. The study subjects that had higher $\gamma$-GT concentration also had lower GPx content, so it was negatively correlated. GPx enzyme is needed by the cell for defense againts stress oxidative by using GSH as a substrate for $\mathrm{H}_{2} \mathrm{O}_{2}$ reduction and lipid peroxide to become a form of safe alcohol (12).

Extracellular breakdown of GSH by $\gamma$-GT triggers a release of ROS through chemical reaction of CysGly $(6,13)$. CysGly level in the circulation, produced by GSH hydrolysis, shows a significant increase in patients with metabolic syndrome. CysGly is a potent reductor of iron ion on extracellular environment and a producer of $\mathrm{Fe} 2$ that has the ability to trigger ROS production. ROS will initiate the process of LDL oxidation. Oxidated LDL components will be a factor that mediate $\gamma$-GT enzyme activity increase with atherogenesis incident (14).

The results of this study also showed that an increase in $\gamma$-GT concentration was negatively correlated with TAS concentration, although not statistically significant. Since TAS concentration implies antioxidant condition in general in the body, it strengthens our assumption that an increase in $\gamma$-GT concentration is related to oxidative stress and a decrease in antioxidant, particularly since oxidative stress is caused by an increase in pro-oxidant enzyme activity level and/or a decrease in antioxidant enzyme activity level.

$\gamma$-GT is stated to have positive correlation with several risk factors for cardiovascular diseases, such as C-Reactive Protein (CRP), Fibrinogen, F2-Isoprostane, and negatively correlated to antioxidant level (8). This study also indicated that an increase in $\gamma$-GT activity level is positively correlated with hsCRP, where the group of higher $\gamma$-GT activity shows higher hsCRP which also means higher risk for cardiovascular diseases.

$\gamma$-GT's itself is stated to be not only a proatherogenic compound that contributes to extracellular GSH metabolism, but also as an enzyme that is found on atherosclerosis plaques. It is suspected as a contributor for its progression and hence its rupture. Eventhough the exact accumulation mechanism of $\gamma$-GT in the atheroscelerosis plaques is still unknown, one strongly suspected mechanism is through lipoprotein influx (15). This suspicion is based on the tendency of lipoprotein LDL of being a $\gamma$-GT carrier to enter into the plaques and also as a free iron ion. Pro-inflammatory and pro-atherogenic nature of lipoprotein LDL creates an even stronger base for its suspicion on this mechanism (16).

Patients experiencing $\gamma$-GT concentration increase has the risk of decrease in GPx content and antioxidant in general. This data strengthens the fact of negative correlation between an increase in $\gamma$-GT activity with GPx and explains that GPx has a protective effect to $\gamma$-GT. The negative correlation is also shown on $\gamma$-GT correlation with TAS, although considered as having a weak significance. Relationship between $\gamma$-GT with several antioxidants such as $\alpha$-carotene, $\beta$-carotene, $\beta$-criptoxantin, zeasantin/ lutein, lycopene, and vitamin $\mathrm{C}$ is stated to be negatively correlated as well. This, however, is a different case with vitamin $\mathrm{E}$, which is stated as having no relationship at all with $\gamma$-GT concentration (1).

Another study done by Arifin M, et al. found that Conorary Heart Disease patients with stable condition and high ratio $\gamma$-GT /GPx comes with a risk of 8.593 times higher with a rise of hsCRP concentration in comparison to Conorary Heart Disease patients with a normal level of hsCRP. Data obtained from this study also indicated a similar risk factor for the metabolic syndrome patients. An increase of $\gamma$-GT concentration in metabolic syndrome 
patients has a risk factor of 8.5 times higher to an increase in hsCRP level in patients. A rise in concentration of $\gamma$-GT can cause a secondary low grade hepatic inflammation that associates with hepatic steatosis $(15,17)$. In metabolic syndrome, there is an increase of IL- 6 production by linkage visceral adipose that eventually triggers CRP secretion by the heart that leads to inflammation (18).

The results of the analyses showed the correlation of $\gamma$-GT/GPx ratio and $\gamma$-GT/TAS ratio with hsCRP with an odds ratio of 7.143 and 4.82 , respectively. The ratio of $\gamma$-GT/GPx ratio and $\gamma$-GT/TAS indicated the oxidative stress where there was an imbalance between ROS and antioxidant. This odds ratio was still below the odds ratio of correlation between $\gamma$-GT and hsCRP. This lower odds ratio explains that a rise in $\gamma$-GT concentration alone is closely related to the rise in hs-CRP concentration on the basis of that ratio. Explanation proposed is that an increase in $\gamma$-GT is more significantly dominant in the inflammatory condition through the GSH catabolics that results GysGly compound with its pro-oxidant and its activity on the leucotrient proinflammatory mediator $(14,19)$.

The result data obtained from this study showed a positive correlation of the number of metabolic syndrome components with GPx content. It is suspected that metabolic syndrome increases free radicals and the body is trying to balance itself by forming antioxidant compounds. Antioxidant enzyme normally increases its production as a response to the increase of ROS (20). All patients have metabolic syndrome before they get fatty liver, so it is suspected that compensation mechanism is still valid.

As the results data obtained from this research are all presented above, it is also important to note that this research was designed as cross-sectional type of study with its various limitations. As such, further studies need to be done, especially with patients having fatty liver included as the study subjects for comparison. This comparison is needed inorder that the distinction of $\gamma$-GT as a sign for oxidative stress and $\gamma$-GT as a sign for liver function would be clearly clarified. A further study using other antioxidant enzyme such as Super Oxide Dismutase (SOD) is needed so that the exact roles of $\gamma$-GT can be explained in more detail.

An increase of $\gamma$-GT concentration in metabolic syndrome patients has a risk factor of 8.5 times higher to an increase in hsCRP level on patients. A rise in concentration level of $\gamma$-GT can cause a secondary inflammation low grade hepatic that associates with hepatic steatosis (15). In metabolic syndrome, an increase of IL- 6 production by linkage visceral adiposeso that it push the CRP secretion by heart that points on inflammation (18)

The result of analysis shows correlation $\gamma$-GT/GPx ratio and $\gamma$-GT/TAS ratio with hsCRP comes with an odd ratio of 7.143 and 4.82 , respectively. The ratio of $\gamma$-GT/ GPx ratio and $\gamma$-GT/TAS depict the oxidative stress where there is an imbalance between ROS and antioxidant. This odd ratio is still below the odd ratio of correlation between $\gamma$-GT and hsCRP. This lower odd ratio explains that a rise in $\gamma$-GT concentration alone is tightly related to a rise in hsCRP concentration on the basis of that ratio. Explanation proposed is that an increase in $\gamma$-GT is more significantly dominant in the inflammatory condition through the GSH catabolics that results GysGly compound with its pro-oxidant and its activity on the leucoctrient proinflammatory mediator $(14,19)$

The result data obtained from this study showed a positive correlation of the number of metabolic syndrome components with GPx content. It is suspected that metabolic syndrome increases free radicals and the body is trying to balance itself by forming antioxidant compounds. Antioxidant enzyme normally increases its production as a response to the increase of ROS (20). All patients have metabolic syndrome before they get fatty liver, so it is suspected that compensation mechanism is still valid.

As the results data obtained from this research are all presented above, it is also important to note that this research was designed as cross-sectional type of study with its various limitations. As such, further studies need to be done, especially with patients having fatty liver included as the study subjects for comparison. This comparison is needed inorder that the distinction of $\gamma$-GT as a sign for oxidative stress and $\gamma$-GT as a sign for liver function would be clearly clarified. A further study using other antioxidant enzyme such as Super Oxide Dismutase (SOD) is needed so that the exact roles of $\gamma$-GT can be explained in more detail.

\section{Conclusions}

1. A rise in $\gamma$-GT enzyme concentration has a negative correlation with GPx enzyme. $\gamma$-GT enzyme also has the tendency to be negatively correlated with TAS, although no statistical data show any significance. We can therefore conclude that a rise in $\gamma$-GT concentration may be used as a sign of a decrease in GPx content and a sign of oxidative stress condition.

2. $\gamma$-GT enzyme concentration has a positive correlation with hsCRP levels. Hence, an increase in $\gamma$-GT concentration might be used as a sign of metabolic 
syndrome patients having proinflammatory and cardiovascular risk factor.

3. A rise in $\gamma$-GT concentration might have a more dominant role in inflammatory condition in comparison to GPx and TAS content in metabolic syndrome patients.

\section{Acknowledgement:}

This study was fully funded by The Prodia Education and Research Institute. We thank the technical staff of Clinical Laboratory for their valuable assistance in undertaking specimen collection and for the biochemical assesments.

\section{Conflict of Interest:}

There is no conflict of interest related to this study.

\section{References:}

1. Lim JS, Yang YH, Chun BY, Kam S, Jacobs DR Jr, Lee DH. Is Serum Gamma-Glutamyltransferase Inversely Associated with Serum Antioxidants as A Marker of Oxidative Stress? Free Radic Biol Med 2004; 37: 1018-2023.

2. Giral P, Jacob N, Dourmap C, Hansel B,Carrie A, Bruckert E, et al., Elevated Gamma-glutamyltranferase Activity and Perturbed Thiol Profile are Assosiated With features of Metabolic Syndrome. Arterioscler Thromb Vasc Biol 2008; 28: 587-93.

3. Bruckert E, Giral P, Ratziu V, Poynard T, Chapman MJ, Opolon $\mathrm{P}$, et al. A constellation of Cardiovascular Risk Factor is Associated with Hepatic Enzyme Elevation in Hyperlipidemic Patient. Metabolism 2002; 51: 1071-6.

4. Paolicchi A, Emdin M, Ghliozeni E, Ciancia E, Passino C, Popoff $\mathrm{G}$, et al. Human Atherosclerotic Plaques Contain Gammaglutamyltranspeptidase Enzyme Activity. Circulation 2004; 109: 1440 .

5. Pompella A, Emdin M, Passino C, Paolicchi A. The Significance of Serum Gamma-Glutamyltransferase in cardiovascular Disease. Clin Chem Lab Med 2004; 42: 1085-91.

6. Accaoui MJ, Enoui MM, Masson, Dominici, Wellman, Visvikis. Gamma-glutamyltranspeptidase-dependent glutathione Catabolism Result in Activation of NF-kB. Biochem Biophys Res Commun 2000; 276 : 1062-7.

7. Ruttmann E, Brant LJ, Concinm H, Diem G, Rapp K, Ulmer H.
Gamma-glutamyltransferase as a Risk Factor for Chronic Heart Disease Mortality: an Epidemiological Investigation in a Cohort of 163944 Australian Adult. Circulation 2005; 112 : 2130-7.

8. Lee Douglas S, Jane C Evans, Sander J Robins, Peter W Wilson, Irene Albano Caroline S Fox, et al. Gamma Glutamyl Transferase and Metabolic Syndrome, Cardiovascular Disease, and Mortality Risk: The Framingham Heart Study, Arterioscler. Thromb. Vasc. Biol. 2007; 27: 127-33.

9. Heistad DD. Oxidative Stress and Vascular Disease. Arterioscler. Thromb. Vasc. Biol 2006; 26: 689-95

10. Lee DH, Jacobs DR Jr, Gross M, Kiefe Cl, Roseman J, Lewis $\mathrm{CE}$, et al. $\mathrm{y}$-Glutamyltransferase is a Predictor of Incident Diabetes and Hypertension: The Coronary Artery Risk Development in Young Adults (CARDIA) Study. Clin Chem 2003; 49: 1358-66.

11. Karp DR, Shimooku K,Lipsky PE. Expression of $y$-Glutamyl Transpeptidase Protection Ramos B Cells from OxidationInduced Cells Death. J Biol Chem 2001; 276: 3798-804

12. Forgione MA, Cap A, Liao R, Moldovan NI, Eberhardt RT, Lim CC, et al. Heterozygous Cellular Glutathione Peroxidase Deficiency in the Mouse. Circulation 2002;106 : 1154-8.

13. Dominici S, Visvikis A, Pieri L, PaolicchiA, Valentini MA, Comporti M, Pompella A. Redox Modulation of NF-kappaB Nuclear Translocation and DNA Binding in Metastatic Melanoma : The Role of Endogenous and Gamma-GlutamyltransferaseDependent Oxidative Stres. Tumori 2003; 89 : 426-33.

14. Paolicchi A, Minotti G, Tonarelli P, Tongiani R De Cesare D, MezzettiA,DominiciS, et al.Gamma-Glutamyltranspeptidase Dependent Iron Reduction and LDL Oxidation a Potential mechanism in Atherosclerosis, J Invest Med 1999; 47: 151 60.

15. Grundy SM. Gamma-Glutamyltransferase Another Biomarker for Metabolic Syndrome and Cardiovascular Risk. Arterioscler Thromb Vasc Biol 2007; 27: 4-7.

16. Emdin M, Passio C, Michelassi, Titta F, L'Abbate A, Donato, Pompella A, Paolicchi A. Prognostic Value of Serum Gamma - Glutamyl Transferase Activity After Myocardial Infarction. Eur Heart J 2001; 22: 1802-7.

17. Arifin $M$, Donosepoetro $M$, Kasiman $S$, Relationship between $\mathrm{Y}$-Glutamyltransferase ( $\mathrm{Y}$-GT) with High Sensitive C-Reactive Protein (hsCRP), Oxidized LDL (Ox-LDL) and Glutathione Peroxidase on Coronary Heart Disease (CHD) Patient. Ina Biomed J 2009; 2 : 51-8.

18. Mathieu $P$, Poirier $P$, Pibarot $P$, Lemieux I, Després JeanPierre. Visceral Obesity: The Link Among Inflammation, Hypertension, and Cardiovascular Disease. Hypertension 2009; 53: 577-84.

19. Bäck, Magnus. Gamma-glutamyltransferase, Leukotrienes, and Cardiovascular Risk. Eur Heart J. 2006; 28: 269-70

20. Madamanchi NR, Vendrov A, Runge MS. Oxidative Stress and Vascular Disease. Arterioscler. Thromb. Vasc. Biol. 2005; 25: 29-38. 